\title{
Translating Classical Probability Logics into Modal Fuzzy Logics
}

\author{
Paolo Baldi ${ }^{a, b}$, Petr Cintula ${ }^{a}$ and Carles Noguera ${ }^{c}$ \\ ${ }^{a}$ Institute of Computer Science \\ Czech Academy of Sciences \\ cintula@cs.cas.cz \\ ${ }^{b}$ Department of Philosophy \\ University of Milan \\ paolo.baldi@unimi.it \\ ${ }^{c}$ Institute of Information Theory and Automation \\ Czech Academy of Sciences \\ noguera@utia.cas.cz
}

\begin{abstract}
This paper is a contribution to the study of two distinct kinds of modal logics for modeling uncertainty. Both approaches use logics with a two-layered syntax, but while one employs classical logic on both levels [6], the other involves a suitable system of fuzzy logic in the upper layer [9]. We take two prominent examples of the former approach, probability logics $\operatorname{Pr}_{l i n}$ and $\operatorname{Pr}_{p o l}$, and build explicit faithful translations into, respectively, the two-layered modal fuzzy logics given by Łukasiewicz logic with $\triangle$ and its expansion with the product connective. We first prove the faithfulness of both translations using semantics of all four involved logics. Then, we use the axiomatization of $\operatorname{Pr}_{l i n}$ and a hypersequent presentation of the two-layered system over Łukasiewicz logic to obtain an alternative syntactical proof.
\end{abstract}

Keywords: Mathematical Fuzzy Logic, Logics of uncertainty, Łukasiewicz logic, Probability logics, Two-layered modal logics.

\section{Introduction}

In recent years, numerous logical systems have been introduced to cope with reasoning about uncertain events. Among them, two of the most prominent examples are the modal logics introduced in [6] (see also [11]), which we denote here as $\operatorname{Pr}_{l i n}$ and $\operatorname{Pr}_{\text {pol }}$. The atomic statements of these logics express linear (resp. polynomial) inequalities of probabilities of classical events and are then combined using classical connectives. Both logics are introduced semantically using Kripke frames enriched by a probability measure, which allows for expressing validity of atomic statements of these logics on events construed as sets of worlds described by classical formulas. Despite dealing with the intrinsically graded notion of probability, the semantics of these logics remains essentially bivalent.

An alternative approach uses the framework of Mathematical Fuzzy Logic and takes sentences like " $\varphi$ is probable" at face value, identifying its truth degree with the probability of $\varphi$; then one combines such formulas using connectives of a suitable fuzzy logic. Hence, this approach shifts the syntactical complexity of the atomic statements of the previous approach to the semantics of the fuzzy logic in question. Such idea was proposed in $[9,10]$ and later studied by numerous authors; see [7] for a thorough survey or [3] for an abstract treatment. In this paper, we consider three different fuzzy logics to govern the behavior of modal formulas: Łukasiewicz logic $€$, its expansion $\mathrm{E}_{\triangle}$ with BaazMonteiro operator $\triangle$, and its further expansion $\mathrm{PE}_{\triangle}$ by product conjunction. The resulting modal logics are denoted here as $\operatorname{Pr}_{€}, \operatorname{Pr}_{€_{\Delta}}$, and $\operatorname{Pr}_{\mathrm{P}_{\triangle}}$ respectively. $^{1}$

We describe some strong formal links between systems of the two kinds and thus show that both approaches are much more closely related than it might seem at first sight. First, in Section 2, we introduce the syntax and semantics of all the logics under investigation. Then, in Section 3, we present translations of $\operatorname{Pr}_{l i n}$ and $\mathrm{Pr}_{p o l}$ into, respectively the logics $\mathrm{Pr}_{\mathrm{E}_{\triangle}}$ and $\mathrm{Pr}_{\mathrm{P} €_{\triangle}}$ with a simple semantical proof of their faithfulness. Section 4 presents an extension of $\operatorname{Pr}_{£}$ into its hypersequent variant $\mathrm{HPr}_{£}$ which is then faithfully translated into a hypersequent calculus $\mathrm{H}$ of relations for Łukasiewicz logic [16], thus providing an alternative proof system for $\operatorname{Pr}_{£}$. In Section 5, we use the results of the previous section to provide a faithful translation of the logic $\operatorname{Pr}_{\text {lin }}$ into $\operatorname{HPr}_{£}$ (and consequently also into $\operatorname{Pr}_{\mathrm{E}_{\triangle}}$ ) without using the crucial results of [6] showing that an axiomatic system proposed there is

\footnotetext{
${ }^{1}$ Let us note that the logics introduced above are known under different names in the literature of their respective communities. We have opted here for a uniform but neutral terminology for ease of reference through the paper.
} 
an axiomatization of $\operatorname{Pr}_{l i n}$. We only assume that it is sound w.r.t. the semantics (which is easy to check) and actually obtain the fact that it indeed is an axiomatization of $\operatorname{Pr}_{l i n}$ as a corollary of our translation. Finally, in Section 6 we add some concluding remarks and hints at future research directions.

\section{Classical and Fuzzy logics of Uncertainty}

\subsection{Propositional core}

In this work we need four propositional logics: (1) classical logic CL casted in the language with the truthconstant $\perp$ and implication $\rightarrow$, (2) Eukasiewicz logic $€$ in the same language, (3) $£_{\triangle}$, the expansion of $€$ in the language with an additional unary connective $\triangle$ known as Baaz-Monteiro projection, and finally (4) $\mathrm{PE}_{\triangle}$, the expansion of $\mathrm{E}_{\triangle}$ with an additional binary connective $\odot$ (called product conjunction). Next, we review some of the properties of these logics needed for the paper; we refer the reader to the corresponding chapters of [2] for more details and references.

We expect the reader to be familiar with the notion of formula (over an arbitrary propositional language) and the notion of evaluation in classical logic. In the case of $\mathrm{E}, \mathrm{E}_{\triangle}$, and $\mathrm{P}_{\triangle}$, (standard) evaluations are functions from the corresponding set of formulas into the real unit interval $[0,1]$, such that $e(\perp)=0$ and

$$
\begin{aligned}
e(\varphi \rightarrow \psi) & =\min \{1,1-e(\varphi)+e(\psi)\} \\
e(\triangle \varphi) & = \begin{cases}1 & \text { if } e(\varphi)=1 \\
0 & \text { otherwise }\end{cases} \\
e(\varphi \odot \psi) & =e(\varphi) \cdot e(\psi)
\end{aligned}
$$

Whenever necessary to avoid confusions, we use subscript $€$ to mark the corresponding connectives and distinguish them from the classical ones.

Let $L$ be any of these four logics. We say that a formula $\varphi$ is the semantical consequence of a set of formulas $\Gamma$ of $L$, in symbols $\Gamma=_{L} \varphi$, if for each evaluation $e$ such that $e(\gamma)=1$ for each $\gamma \in \Gamma$ we have $e(\varphi)=1$.

We expect the reader to be familiar with the notion of derivability in a Hilbert-style axiomatic system $\vdash_{A X}$; we say that $A X$ is an axiomatization of a logic $L$ if for each finite set $\Gamma \cup\{\varphi\}$ of formulas, we have: $\Gamma=_{L} \varphi$ iff $\Gamma \vdash_{A X} \varphi$. It is well known that there are numerous axiomatizations of both the classical logic (where the equivalence holds even for infinite sets of premises) and the three fuzzy logics considered here. We write $\vdash_{L}$, when an axiomatization of a logic $L$ is fixed or known from the context.

Let us conclude this subsection by recalling additional definable connectives of $€$ together with their definition and standard semantics:

$$
\begin{array}{lll}
\neg \varphi & \varphi \rightarrow \perp & 1-x \\
\varphi \vee \psi & (\varphi \rightarrow \psi) \rightarrow \psi & \max \{x, y\} \\
\varphi \wedge \psi & \neg(\neg \varphi \vee \neg \psi) & \min \{x, y\} \\
\varphi \oplus \psi & \neg \varphi \rightarrow \psi & \min \{1, x+y\} \\
\varphi \otimes \psi & \neg(\neg \varphi \oplus \neg \psi) & \max \{0, x+y-1\} \\
\varphi \ominus \psi & \neg(\varphi \rightarrow \psi) & \max \{0, x-y\} .
\end{array}
$$

\subsection{Five two-layered modal languages}

We start by recalling the language $\mathcal{L}^{\mathrm{Pr}_{\text {lin }}}$ of the logic $\mathrm{Pr}_{\text {lin }}$. It is a two-layered modal language: first, in a lower layer, we have the non-modal formulas which are simply those of classical propositional logic. We have then basic inequality formulas of the form

$$
\sum_{i=1}^{n} a_{i} P\left(\varphi_{i}\right) \geq c
$$

for $\varphi_{i}$ s being non-modal formulas and $c$ and $a_{i}$ being constants for integers (real numbers are used in a similar system presented in [11], rationals in [5]). The linear combination on the left-hand side of the inequality is called basic inequality term. The formulas of the upper layer of $\mathcal{L}^{\operatorname{Pr}_{l i n}}$, called modal formulas, are then obtained from basic inequality formulas via the usual connectives of classical logic. Obvious abbreviations apply; in particular, we use the following:

$$
\begin{array}{rll}
-\sum_{i=1}^{n} a_{i} P\left(\varphi_{i}\right) & \text { for } \quad \sum_{i=1}^{n}-a_{i} P\left(\varphi_{i}\right) \\
P(\varphi) \geq P(\psi) & \text { for } \quad P(\varphi)-P(\psi) \geq 0 \\
t \leq c & \text { for } \quad-t \geq-c \\
t<c & \text { for } \quad \neg(t \geq c) \\
t=c & \text { for } \quad(t \geq c) \wedge(t \leq c) .
\end{array}
$$

The language $\mathcal{L}^{\operatorname{Pr}_{\text {pol }}}$ is obtained by using again the language of classical logic for the lower layer, and allowing any polynomial basic inequality terms in the upper layer, i.e., the basic inequality formulas of $\mathcal{L}^{\operatorname{Pr}_{\text {lin }}}$ have the general form

$$
\sum_{i=1}^{n} a_{i} P\left(\varphi_{i}^{1}\right) \cdots P\left(\varphi_{i}^{m_{i}}\right) \geq c .
$$

Complex formulas of the upper layer are built as for $\mathcal{L}^{\operatorname{Pr}_{l i n}}$, combining basic inequality formulas by the connectives of classical logic. Note that in $\mathcal{L}^{\operatorname{Pr}_{p o l}}$ one can express fundamental probabilistic notions, e.g. independence of events using formulas of the kind $P(\varphi \wedge \psi)=P(\varphi) \cdot P(\psi)$.

Let us now turn our attention to the fuzzy approach towards logics of probability. We introduce three languages, $\mathcal{L}^{\mathrm{Pr}_{\mathrm{E}}}$ (resp. $\mathcal{L}^{\mathrm{Pr}_{\mathrm{E}}}$ or $\left.\mathcal{L}^{\mathrm{Pr}_{\mathrm{PE}}}\right)$ ), where, as before, the lower-layer formulas are those of classical logic, but instead of basic inequality formulas combined 
by connectives of classical logic, the modal formulas are built from simple atomic modal formulas of the form $P(\varphi)$ (for $\varphi$ being classical formula) using the connectives of the corresponding logic.

Remark 1. Note that a basic inequality formula $\sum_{i=1}^{n} a_{i} P\left(\varphi_{i}\right) \geq c$ of $\mathcal{L}^{\operatorname{Pr}_{\text {lin }}}$ can be seen as an atomic modal formula obtained by applying an $n$-ary modality $\square_{a_{1}, \ldots, a_{n}, c}$, on $n$ classical formulas $\varphi_{1}, \ldots, \varphi_{n}$. In this way, one can see $\mathcal{L}^{\operatorname{Pr}_{\text {lin }}}$ as an instance of an abstract two-layered modal language in the sense of [3]. The same is true for $\mathcal{L}^{\mathrm{Pr}_{\text {pol }}}$, but here the set of used modalities is even more complex. Thus, the five languages can be summarized in the following table:

\begin{tabular}{llll} 
Language & Lower $l$. & Modalities & Upper $l$. \\
\hline $\mathcal{L}^{\operatorname{Pr}_{\text {lin }}}$ & $\mathrm{CL}$ & $\{t \geq c: t$ lin $\}$ & $\mathrm{CL}$ \\
$\mathcal{L}^{\operatorname{Pr}_{\text {pol }}}$ & $\mathrm{CL}$ & $\{t \geq c: t$ poly $\}$ & $\mathrm{CL}$ \\
$\mathcal{L}^{\operatorname{Pr}_{\mathrm{E}}}$ & $\mathrm{CL}$ & $\{P\}$ & $\mathrm{E}$ \\
$\mathcal{L}^{\mathrm{Pr}_{\mathrm{E}}}$ & $\mathrm{CL}$ & $\{P\}$ & $\mathrm{E}_{\triangle}$ \\
$\mathcal{L}^{\mathrm{Pr}_{\mathrm{PE}}}$ & $\mathrm{CL}$ & $\{P\}$ & $\mathrm{PE}_{\triangle}$
\end{tabular}

\subsection{One semantics and five logics}

The semantical picture for all five languages is based on Kripke models enriched by (finitely additive) probability measures. A (probabilistic) Kripke model is a triple $\mathbf{M}=\left\langle W,\left\langle e_{w}\right\rangle_{w \in W}, \mu\right\rangle$, where

- $W$ is a non-empty set of worlds,

- $e_{w}$ s are classical propositional evaluations,

- $\mu$ is a finitely additive measure over a Boolean subalgebra of the powerset algebra of $W$ such that

$$
\varphi^{\mathbf{M}}=\left\{w: e_{w}(\varphi)=1\right\}
$$

is a measurable set for any classical formula $\varphi$.

Clearly, $\mathbf{M}$ allows us to define the truth values of nonmodal formulas in each of its worlds. The assignment of truth values of modal formulas depends on the language in question, but in all cases we evaluate modal formulas only at the level of the whole model.

For the basic inequality formulas of $\mathcal{L}^{\operatorname{Pr}_{\text {lin }}}$ we define:

$$
\left\|\sum_{i=1}^{n} a_{i} P\left(\varphi_{i}\right) \geq c\right\|_{\mathbf{M}}=1 \quad \text { iff } \quad \sum_{i=1}^{n} a_{i} \mu\left(\varphi_{i}{ }^{\mathbf{M}}\right) \geq c .
$$

The truth values of basic inequality formulas of $\mathcal{L}^{\operatorname{Pr}_{\text {pol }}}$ are defined analogously, and truth values of complex modal formulas in both languages are then defined using the truth-functions of classical connectives.

We recall that $\mathcal{L}^{\mathrm{Pr}_{\mathrm{E}}}, \mathcal{L}^{\mathrm{Pr}_{\mathrm{E}}} \Delta$, and $\mathcal{L}^{\mathrm{Pr}_{\mathrm{PE}}}$ share the same atomic modal formulas and define their truth values simply as:

$$
\|P(\varphi)\|_{\mathbf{M}}=\mu\left(\varphi^{\mathbf{M}}\right) .
$$

Then, clearly, we always have $\|P(\varphi)\|_{\mathbf{M}} \in[0,1]$, and so we can compute the truth values of more complex modal formulas using truth functions for connectives of the corresponding logic.

For each of the five languages we have introduced, we can define a consequence relation on the corresponding set of modal formulas as preservation of the truth value 1 over all Kripke models; for instance, for each set $\Gamma \cup\{\varphi\}$ of modal $\mathcal{L}^{\operatorname{Pr}_{l i n}}$-formulas, we define:

$$
\begin{array}{r}
\Gamma \models \operatorname{Pr}_{\text {lin }} \varphi \text { iff }\|\varphi\|_{\mathbf{M}}=1 \text { for each Kripke model } \mathbf{M} \\
\text { where }\|\gamma\|_{\mathbf{M}}=1 \text { for each } \gamma \in \Gamma .
\end{array}
$$

Analogously, we define the consequence relations $\models=\operatorname{Pr}_{p o l}, \models \operatorname{Pr}_{E}, \models \operatorname{Pr}_{\triangle}$, and $\models \operatorname{Pr}_{\mathrm{PE}_{\triangle}}$.

\subsection{Axiomatization results}

An axiomatization for $\operatorname{Pr}_{l i n}$ has been presented in [6]. It consists of: (1) any axiomatization of classical logic for both modal and non-modal formulas, (2) the following axioms

$\begin{array}{ll}\text { (QU1) } & P(\phi) \geq 0 \\ \text { (QU2) } & P(\top)=1 \\ \text { (QU3) } & P(\varphi \wedge \psi)+P(\varphi \wedge \neg \psi)=P(\varphi) \\ \text { (QUGEN) } & \text { From } \varphi \leftrightarrow \psi \text { infer } P(\varphi)=P(\psi)\end{array}$

and (3) the axioms to manipulate linear inequalities, meant to be instantiated with any basic inequality formulas $\sum_{i=1}^{k} a_{i} P\left(\varphi_{i}\right) \geq c$, integers $d^{\prime}<c$ and $d>0$ and permutations $\sigma$ :

$$
\begin{aligned}
& \text { (LQ1) } P(\varphi) \geq P(\varphi) \\
& \text { (LQ2) } \sum_{i=1}^{k} a_{i} P\left(\varphi_{i}\right) \geq c \leftrightarrow \sum_{i=1}^{k} a_{i} P\left(\varphi_{i}\right)+0 P(\varphi) \geq c \\
& \text { (LQ3) } \sum_{i=1}^{k} a_{i} P\left(\varphi_{i}\right) \geq c \leftrightarrow \sum_{i=1}^{k} a_{\sigma(i)} P\left(\varphi_{\sigma(i)}\right) \geq c \\
& \text { (LQ4) } \sum_{i=1}^{k} a_{i} P\left(\varphi_{i}\right) \geq c \wedge \sum_{i=i}^{k} b_{i} P\left(\varphi_{i}\right) \geq c^{\prime} \rightarrow \\
& \qquad \sum_{i=1}^{k}\left(a_{i}+b_{i}\right) P\left(\varphi_{i}\right) \geq c+c^{\prime} \\
& \text { (LQ5) } \sum_{i=1}^{k} a_{i} P\left(\varphi_{i}\right) \geq c \leftrightarrow \sum_{i=1}^{k} d a_{i} P\left(\varphi_{i}\right) \geq d c \\
& \text { (LQ6) } \sum_{i=1}^{k} a_{i} P\left(\varphi_{i}\right) \geq c \vee \sum_{i=1}^{k} a_{i} P\left(\varphi_{i}\right) \leq c \\
& \text { (LQ7) } \sum_{i=1}^{k} a_{i} P\left(\varphi_{i}\right) \geq c \rightarrow \sum_{i=1}^{k} a_{i} P\left(\varphi_{i}\right)>d^{\prime}
\end{aligned}
$$

The proof that this axiomatic systems is indeed an axiomatization of $\operatorname{Pr}_{\text {lin }}$ relies essentially on linear programming methods. Let us note that there is no axiomatization provided for $\operatorname{Pr}_{p o l}$ in [6], though it is shown to be PSPACE-complete via a reduction to real closed field theory. 
In contrast, the axiomatizations of $\operatorname{Pr}_{€}, \operatorname{Pr}_{\mathrm{PE}_{\triangle}}$, and $\mathrm{Pr}_{\mathrm{P}{ }_{\triangle}}$ are much simpler (see $[3,7,8]$ ). They use any axiomatization of classical logic for non-modal formulas, any axiomatization of $€$ (or $\ell_{\triangle}$ or $\mathrm{P}_{\triangle}$ respectively) for modal formulas and just three additional axioms and one rule:

$$
\begin{array}{ll}
\text { (A1) } & \left(P \varphi \odot_{£} P(\varphi \rightarrow \psi)\right) \rightarrow_{£} P \psi \\
\text { (A2) } & P \neg \varphi \leftrightarrow_{£} \neg_{£} P \varphi \\
\text { (A3) } & P(\varphi \vee \psi) \leftrightarrow_{£}\left[\left(P \varphi \ominus_{£} P(\varphi \wedge \psi)\right) \oplus_{£} P \psi\right]
\end{array}
$$

(Nec) From $\varphi$ infer $P \varphi$.

\section{The translations}

Now we are ready to show that the classical probability logic $\operatorname{Pr}_{l i n}$ can be faithfully translated into the modal fuzzy logic $\operatorname{Pr}_{\mathrm{E}_{\triangle}}$. Let $t \geq c$ be a basic inequality formula in $\mathcal{L}^{\operatorname{Pr}_{l i n}}$, where $t$ stands for $\sum_{i=1}^{n} a_{i} P\left(\varphi_{i}\right)$, and consider the linear polynomial with integer coefficients

$$
f\left(x_{1}, \ldots, x_{n}\right):=\sum_{i=1}^{n} a_{i} x_{i}-c+1 .
$$

By the well-known McNaughton Theorem (see e.g. [1, Lemma 2.1.21]), one can algorithmically build a formula $\gamma$ of $\mathrm{\ell}$ over variables $p_{1}, \ldots, p_{n}$, such that for any standard evaluation $e$ of $€$ we have

$$
e(\gamma)=\max \left\{0, \min \left\{1, f\left(e\left(p_{1}\right), \ldots, e\left(p_{n}\right)\right)\right\}\right\} .
$$

By $(t \geq c)^{\bullet}$ we denote the formula resulting from $\Delta \gamma$ by replacing each variable $p_{i}$ in $\gamma$ by $P\left(\varphi_{i}\right)$.

Clearly, $(t \geq c)^{\bullet}$ is a formula of $\mathcal{L}^{\mathrm{Pr}_{\mathrm{E}}} \Delta$. We can easily extend it to a translation of all modal formulas from $\mathcal{L}^{\operatorname{Pr}_{\text {lin }}}$ by setting $\perp^{\bullet}=\perp_{£}$ and $(\gamma \rightarrow \delta)^{\bullet}=\gamma^{\bullet} \rightarrow_{£} \delta^{\bullet}$. Let us denote by $\Gamma^{\bullet}$ the set resulting from applying the translation to each formula in $\Gamma$.

Theorem 1. Let $\Gamma \cup\{\delta\}$ be a set of modal formulas of $\mathcal{L}^{\operatorname{Pr}_{l i n}}$. Then, $\Gamma \models \operatorname{Pr}_{\text {lin }} \delta$ iff $\Gamma^{\bullet} \models_{\operatorname{Pr}_{\mathrm{E}_{\triangle}}} \delta^{\bullet}$.

Proof. It is easy to see that all we need to prove is that, for each Kripke model $\mathbf{M}$ and each modal formula $\chi$

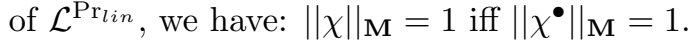

We prove the claim by induction over the complexity of $\chi$. Assume that $\chi$ is a basic inequality formula $\sum_{i=1}^{n} a_{i} P\left(\varphi_{i}\right) \geq c$. Then, we can write the following sequence of equivalences: $\|\chi\|_{\mathbf{M}}=1$ iff $\sum_{i=1}^{n} a_{i} \mu\left(\varphi_{i}{ }^{\mathbf{M}}\right) \geq c$ iff $\sum_{i=1}^{n} a_{i}\left\|P\left(\varphi_{i}\right)\right\|_{\mathbf{M}} \geq c$ iff $\max \left\{0, \min \left\{1, \sum_{i=1}^{n} a_{i}\left\|P\left(\varphi_{i}\right)\right\|_{\mathbf{M}}-c+1\right\}\right\}=1$ iff $\left\|\gamma^{\bullet}\right\|_{\mathbf{M}}=1$.

To prove the induction step, we only need to note that (1) for a basic inequality formula $\chi$ we have that (thanks to the semantics of $\triangle$ ) $\left\|\chi^{\bullet}\right\|_{\mathbf{M}}<1$ implies $\left\|\chi^{\bullet}\right\|_{\mathbf{M}}=0$ and (2) the Eukasiewicz implication behaves on values 0 and 1 as the classical one.
Now we extend the translation to $\mathrm{Pr}_{p o l}$ and $\operatorname{Pr}_{\mathrm{P}} \iota_{\triangle}$. Recall that no axiomatization of $\mathrm{Pr}_{p o l}$ is provided in $[6,11]$ and so, thanks to the known axiomatization of $\mathrm{Pr}_{\mathrm{PE}_{\triangle}}$, this translation can be seen as a first, though indirect, axiomatization of $\operatorname{Pr}_{p o l}$. Let $t \geq c$ be a basic inequality formula in $\mathcal{L}^{\mathrm{Pr}_{\text {pol }}}$ of the form:

$$
\sum_{i=1}^{n} a_{i} P\left(\varphi_{i}^{1}\right) \cdots P\left(\varphi_{i}^{m_{i}}\right) \geq c
$$

As before, we consider the linear polynomial:

$$
f\left(x_{1}, \ldots, x_{n}\right):=\sum_{i=1}^{n} a_{i} x_{i}-c+1,
$$

and the corresponding formula $\gamma$ of $€$ over propositional variables $p_{1}, \ldots, p_{n}$, such that

$$
e(\gamma)=\max \left\{0, \min \left\{1, f\left(e\left(p_{1}\right), \ldots, e\left(p_{n}\right)\right)\right\}\right\} .
$$

Let us by $(t \geq c)^{\bullet}$ denote the formula resulting from $\triangle \varphi$ by replacing each propositional variable $p_{i}$ in $\varphi$ by $P\left(\varphi_{i}^{1}\right) \cdots P\left(\varphi_{i}^{m_{i}}\right)$.

Theorem 2. Let $\Gamma \cup\{\delta\}$ be a set of formula of $\mathcal{L}^{\operatorname{Pr}_{p o l}}$. Then, $\Gamma \models \operatorname{Pr}_{\text {pol }} \delta$ iff $\Gamma^{\bullet} \models \operatorname{Pr}_{\mathrm{PE}_{\triangle}} \delta^{\bullet}$.

\section{An alternative proof system for $\operatorname{Pr}_{€}$}

In this section we extend $\operatorname{Pr}_{€}$ into a hypersequent variant $\operatorname{HPr}_{£}$ which we faithfully translate into a hypersequent calculus HŁ of relations for Łukasiewicz logic [16]. Therefore H£ can be seen as an alternative proof system for $\operatorname{Pr}_{モ}$.

A sequent of relations is a syntactic object of the kind $\Gamma \triangleleft \Delta$ where $\Gamma, \Delta$ are multisets of formulas, and $\triangleleft$ stands for either the symbol $\leq$ or $<$. A hypersequent $G$ is a finite multiset of sequents of relations, denoted as $\Gamma_{1} \triangleleft_{1} \Delta_{1}|\cdots| \Gamma_{n} \triangleleft_{n} \Delta_{n}$, where each sequent of relation $\Gamma_{i} \triangleleft_{i} \Delta_{i}$ belonging to $G$ is called a component of the hypersequent. We refer to hypersequents containing only formulas in the language of Eukasiewicz logic as Ł-hypersequents, and to hypersequents containing formulas in the language of $\operatorname{Pr}_{£}$ as $\operatorname{Pr}_{£}$-hypersequents. Henceforth, we denote by $[\gamma]^{n}$ the multiset composed of $n$ occurrences of $\gamma$.

Let us define the semantics of E-hypersequents and the corresponding consequence relation. First, we extend an evaluation $e$ to multisets of formulas by letting $e(\Gamma)=\sum_{\varphi \in \Gamma}(e(\varphi)-1)$ and $e(\emptyset)=0$, then we say that $e$ satisfies an Ł-hypersquent $G$ if there is a component $\Gamma \triangleleft \Delta$ of $G$ such that $e(\Gamma) \triangleleft e(\Delta)$. By $G_{1}, \ldots, G_{n} \models$ HE $G$ we mean that any evaluation $e$, satisfying $G_{1}, \ldots, G_{n}$, satisfies $G$ as well. Note that $\models_{\mathrm{H}} \emptyset \leq \varphi$ iff $\models_{\mathrm{E}} \varphi$ (indeed, $e(\emptyset) \leq e(\varphi)-1$, iff $0 \leq e(\varphi)-1$, iff $1 \leq e(\varphi)$, that is $\left.=_{\mathrm{E}} \varphi\right)$. 


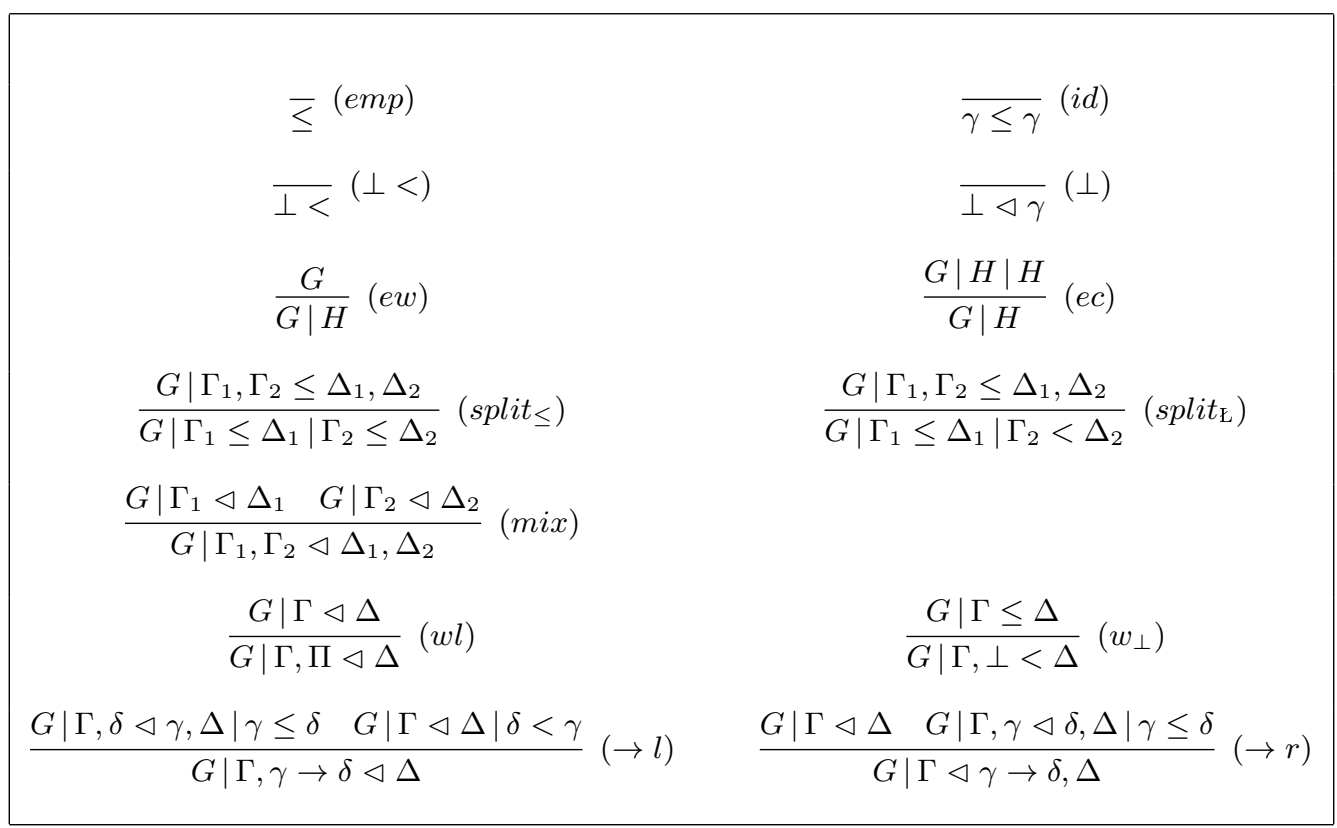

Table 1: Hypersequent calculus of relations HŁ for $\mathrm{L}$

For $\operatorname{Pr}_{\complement}$-hypersequents we proceed analogously: Given a Kripke model $\mathbf{M}$ and a multiset $\Gamma$ of formulas of $\operatorname{Pr}_{£}$, we let $\|\Gamma\|_{\mathbf{M}}=\sum_{\gamma \in \Gamma}\left(\|\gamma\|_{\mathbf{M}}-1\right)$ and say that $\mathbf{M}$ satisfies a $\operatorname{Pr}_{€}$-hypersequent $G$ if $\|\Gamma\|_{\mathrm{M}} \triangleleft\|\Delta\|_{\mathrm{M}}$ for some component $\Gamma \triangleleft \Delta$ of $G$; the consequence relation $G_{1}, \ldots, G_{n}=_{\mathrm{HPr}_{\mathrm{E}}} G$ is then defined as expected. As in the case of $\models_{\mathrm{HE}}$, we have $\models_{\mathrm{HPr}_{\mathrm{E}}} \emptyset \leq \varphi$ iff $\models_{\operatorname{Pr}_{\mathrm{E}}} \varphi$, i.e., $\operatorname{HPr}_{€}$ naturally contains the logic $\operatorname{Pr}_{モ}$.

The hypersequent calculus of relations HE is displayed in Table 1. Recall that a derivation of a hypersequent $G$ from hypersequents $G_{1}, \ldots, G_{n}$ in such calculus is just a labeled tree, where the root is $G$, each node is labelled by the application of a rule of HE and the leaves are either axioms or one of $G_{1}, \ldots, G_{n}$. By $G_{1}, \ldots, G_{n} \vdash_{\mathrm{HE}} G$ we mean that there exists a derivation of $G$ from $G_{1}, \ldots, G_{n}$ in HE.

Now we are ready to define the translation. It can be seen as a hypersequent variant of the translation of $\mathrm{Pr}_{E}$ into $\mathrm{E}$, which is at the core of various proofs of completeness of $\operatorname{Pr}_{\mathrm{E}}$ (the original idea is due to Hájek $[8]$ and is further developed in subsequent works; see e.g. $[3,7])$. First, for any classical formula $\varphi$ we define:

$$
[\varphi]=\left\{\psi: \vdash_{\mathrm{CL}} \psi \leftrightarrow \varphi\right\} .
$$

Now, for any atomic modal formula $P(\varphi)$, we let $P(\varphi)^{*}=p_{[\varphi]}$, where $p_{[\varphi]}$ is a fresh propositional variable in the language of $€$, and for complex modal formulas, we let $\left(\gamma_{1} \rightarrow_{€} \gamma_{2}\right)^{*}=\gamma_{1}^{*} \rightarrow_{€} \gamma_{2}^{*}$ and $\perp_{€}^{*}=\perp_{€}$. We also extend the translation to multisets of formulas in an expected way, i.e. $\left[\gamma_{1}, \ldots, \gamma_{n}\right]^{*}=\left[\gamma_{1}^{*}, \ldots, \gamma_{n}^{*}\right]$. For a hypersequent $G=\Gamma_{1} \triangleleft_{1} \Delta_{1}|\ldots| \Gamma_{n} \triangleleft_{n} \Delta_{n}$ we then define $G^{*}=\Gamma_{1}^{*} \triangleleft_{1} \Delta_{1}^{*}|\cdots| \Gamma_{n}^{*} \triangleleft_{n} \Delta_{n}^{*}$.
Finally, we need to include a translation of the axioms and the rule of $\mathrm{Pr}_{\mathrm{E}}$ into a hypersequent of HE. In order to keep the translation finite we need to make it relative to a given finite set $V$ of propositional variables. Let us define the hypersequent $A X_{V}^{*}$ as the smallest multiset containing all the hypersequents from the following four sets (by $V_{\varphi}$ we denote the set of variables occurring in $\varphi$ ):

$$
\begin{aligned}
R_{V}= & \left\{p_{[\varphi]}<\emptyset: V_{\varphi} \subseteq V, \vdash_{\mathrm{CL}} \varphi\right\} \\
A_{V}^{1}= & \left\{p_{[\psi]}, \perp<p_{[\varphi]}, p_{[\varphi \rightarrow \psi]}: V_{\varphi}, V_{\psi} \subseteq V\right\} \\
A_{V}^{2}= & \left\{p_{[\varphi]}, p_{[\neg \varphi]}<\perp \mid \perp<p_{[\neg \varphi]}, p_{[\varphi]}: V_{\varphi} \subseteq V\right\} \\
A_{V}^{3}=\left\{p_{[\varphi \vee \psi]}, p_{[\varphi \wedge \psi]}<p_{[\varphi]}, p_{[\psi]} \mid\right. & \\
& \left.\quad p_{[\varphi \vee \psi]}, p_{[\varphi \wedge \psi]}<p_{[\varphi]}, p_{[\psi]}: V_{\varphi}, V_{\psi} \subseteq V\right\}
\end{aligned}
$$

Note that the hypersequents above are meant to express the negation of properties of probability. The reason for this will be clear in the following lemma.

Lemma 1. For any $\operatorname{Pr}_{\mathrm{E}}$-hypersequent $G$ in variables from a set $V$, we have: $\models_{\mathrm{HPr}_{\mathrm{E}}} G$ iff $\models_{\mathrm{HE}} G^{*} \mid A X_{V}^{*}$ iff $\vdash_{\mathrm{HE}} G^{*} \mid A X_{V}^{*}$.

Proof. We prove the first equivalence only; the second one follows from the axiomatization of HE, see e.g. [16]. We prove the right-to-left direction counterpositively. Assume that $\forall_{\mathrm{HPr}_{\mathrm{E}}} G$, i.e., there is a Kripke model $\mathbf{M}$ such that, for each $\Gamma \triangleleft \Delta \in G$, we have $\|\Gamma\|_{\mathbf{M}} \Varangle$ $\|\Delta\|_{\mathbf{M}}$. Let now $\hat{e}$ be an evaluation of Lukasiewicz logic such that $\hat{e}\left(p_{[\varphi]}\right)=\|P(\varphi)\|_{\mathbf{M}}$ for each $\varphi$ (it is well defined because if $p_{[\varphi]}=p_{[\psi]}$, then $\vdash_{\mathrm{CL}} \varphi \leftrightarrow \psi$ and so $\left.\|P(\varphi)\|_{\mathbf{M}}=\|P(\psi)\|_{\mathbf{M}}\right)$. It is straightforward to check that $\hat{e}$ does not satisfy any of the components 
of $G^{*} \mid A X_{V}^{*}$, i.e., $\forall_{\mathrm{E}} G^{*} \mid A X_{V}^{*}$ (note that here we have crucially used the fact that components of $A X_{V}^{*}$ are defined in a 'negative' fashion).

For the left-to-right direction, assume that $\hat{e}$ is a countermodel of $G^{*} \mid A X_{V}^{*}$, i.e. $\hat{e}\left(\Gamma^{*}\right) \not \hat{e}\left(\Delta^{*}\right)$ for each component $\Gamma^{*} \triangleleft \Delta^{*} \in G^{*}$ and $\hat{e}$ is a countermodel of $A X_{V}^{*}$. The latter means that, for each $\varphi, \psi$ with $V_{\varphi}, V_{\psi} \subseteq V$ : (1) $\hat{e}\left(p_{[\psi]}\right) \geq \hat{e}\left(p_{[\varphi]}\right)+\hat{e}\left(p_{[\varphi \rightarrow \psi]}\right)$, (2) $\hat{e}\left(p_{[\neg \varphi]}\right)=1-\hat{e}\left(p_{[\varphi]}\right),(3) \hat{e}\left(p_{[\varphi \vee \psi]}\right)+\hat{e}\left(p_{[\varphi \wedge \psi]}\right)=$ $\hat{e}\left(p_{[\varphi]}\right)+\hat{e}\left(p_{[\psi]}\right)$ and $(4) p_{[\top]} \geq 1$.

Let $W$ be the set of classical evaluations and consider the subset of the powerset of $W$ defined as:

$$
B_{V}=\left\{\{w: w(\varphi)=1\}: \varphi \text { a formula and } V_{\varphi} \subseteq V\right\} .
$$

Clearly, $B_{V}$ is the domain of a Boolean subalgebra $\boldsymbol{B}_{V}$ of the powerset algebra of $W$. Then, we define the function $\mu^{\prime}: B_{V} \rightarrow[0,1]$ as $\mu^{\prime}(\{w: w(\varphi)=$ $1\})=\hat{e}\left(p_{[\varphi]}\right)$. Due to the properties (1)-(4) above, we know that $\mu^{\prime}$ is a finitely additive probability measure on $\boldsymbol{B}_{V}$ and so, by Horn-Tarski theorem [13] (see as well [14, Theorem 6]), we know that there is a finitely additive probability measure $\mu$ on the powerset algebra of $W$ such that $\mu(X)=\mu^{\prime}(X)$ for each $X \in B_{V}$. Then, $\mathbf{M}=\left\langle W,\langle w\rangle_{w \in W}, \mu\right\rangle$ is a Kripke model (the measurability condition is trivial as all subsets of $W$ are $\mu$-measurable) and we only need to check that $\mathbf{M}$ is a countermodel for $G$. This is a routine check, since $\|P(\varphi)\|_{\mathbf{M}}=\hat{e}\left(p_{[\varphi]}\right)$ and hence $\|\Gamma\|_{\mathbf{M}}=\hat{e}\left(\Gamma^{*}\right)$ for each multiset $\Gamma$ of modal formulas occurring in $G$.

\section{Translation of $\operatorname{Pr}_{l i n}$ into $\mathrm{HPr}_{\mathrm{E}}$}

In this section, we show a faithful translation of the logic $\operatorname{Pr}_{\text {lin }}$ into $\mathrm{HPr}_{\mathrm{E}}$ (and consequently also into $\operatorname{Pr}_{\mathrm{E}_{\triangle}}$ ) without using the fact that the proof system presented in Subsection 2.4 is an axiomatization of $\operatorname{Pr}_{l i n}$. We only assume the soundness of this axiomatic system w.r.t. the semantics of $\operatorname{Pr}_{l i n}$, i.e., if there is a proof of $\varphi$ from premises $\Gamma$, then $\Gamma=\operatorname{Pr}_{\text {lin }} \varphi$, which is easy to check. We actually obtain the converse direction (i.e., the fact that it indeed is an axiomatization of $\operatorname{Pr}_{l i n}$ ) as a corollary of our translation.

We use the fact that any modal formula of $\operatorname{Pr}_{l i n}$ is equivalent to a conjunction of clauses (i.e., disjunctions of literals, in our case basic inequality formulas and their negations) and define the translation $(\cdot)^{H}$ of clauses of $\operatorname{Pr}_{l i n}$ into $\operatorname{Pr}_{€}$-hypersequents.

Let us start by basic inequality formulas. Note that an inequality $t \geq c$ for $t=\sum_{i=1}^{n} a_{i} P\left(\varphi_{i}\right)$ can be equivalently replaced (modulo a suitable permutation) by

$$
\sum_{i=1}^{m} a_{i} P\left(\varphi_{i}\right) \leq \sum_{i=m+1}^{n} a_{i} P\left(\varphi_{i}\right)-c
$$

where all the $a_{i}$ s are positive. Thus we can define:

$$
\begin{gathered}
\Gamma_{t \geq c}=\left[P\left(\varphi_{1}\right)\right]^{a_{1}}, \ldots,\left[P\left(\varphi_{m}\right)\right]^{a_{m}} \\
\Delta_{t \geq c}=\left[P\left(\varphi_{m+1}\right)\right]^{a_{m+1}}, \ldots,\left[P\left(\varphi_{n}\right)\right]^{a_{n}} \\
s(t \geq c)=\sum_{i=1}^{m} a_{i}-\sum_{i=m+1}^{n} a_{i}+c \\
(t \geq c)^{H}= \begin{cases}\Gamma_{t \geq c} \leq \Delta_{t \geq c},[\perp]^{s(t \geq c)} & \text { if } s(t \geq c) \geq 0 \\
\Gamma_{t \geq c},[\perp]^{-s(t \geq c)} \leq \Delta_{t \geq c} & \text { otherwise. }\end{cases}
\end{gathered}
$$

Analogously, we rewrite the inequality $\neg(t \geq c)$ as

$$
\sum_{i=1}^{m} a_{i} P\left(\varphi_{i}\right)<\sum_{i=m+1}^{n} a_{i} P\left(\varphi_{i}\right)+c
$$

so that all $a_{i}$ s are positive; we define $\Gamma_{t<c}$ and $\Delta_{t<c}$ as before, and let

$$
\begin{aligned}
& s(t<c)=\sum_{i=1}^{m} a_{i}-\sum_{i=m+1}^{n} a_{i}-c \\
& (\neg(t \geq c))^{H}= \begin{cases}\Gamma_{t<c}<\Delta_{t<c},[\perp]^{s(t<c)} & \text { if } s(t<c) \geq 0 \\
\Gamma_{t<c},[\perp]^{-s(t<c)}<\Delta_{t<c} & \text { otherwise. }\end{cases}
\end{aligned}
$$

Finally, given any clause $\delta=\gamma_{1} \vee \cdots \vee \gamma_{n}$ in $\mathcal{L}^{\operatorname{Pr}_{l i n}}$, we let $\delta^{H}$ be the hypersequent $\gamma_{1}^{H}|\cdots| \gamma_{n}^{H}$.

Lemma 2. Let $\delta$ be a clause in $\mathcal{L}^{\operatorname{Pr}_{\text {lin }}}$ such that $=\operatorname{Pr}_{\text {lin }} \delta$. Then $\models \operatorname{HPr}_{\mathrm{E}} \delta^{H}$.

Proof. We show that any model $\mathbf{M}$ of $\delta$ is a model of $\delta^{H}$. Let $\delta=\gamma_{1} \vee \cdots \vee \gamma_{n}$, with $\gamma_{i}$ literals, be of the form $\sum_{k=1}^{n_{i}} a_{i k} P\left(\varphi_{i k}\right) \geq c_{i}$ or $\neg\left(\sum_{k=1}^{n_{i}} a_{i j} P\left(\varphi_{i k}\right) \geq c_{i}\right)$ and let $v_{i k}=\mu\left(\varphi_{i k}^{\mathbf{M}}\right)$. Recall the definition of the translation $(\cdot)^{H}$ : for each literal $\gamma_{i}$, the sequent $\gamma_{i}^{H}$ is either of the form

$$
\Gamma_{\gamma_{i}} \triangleleft \Delta_{\gamma_{i}},[\perp]^{s\left(\gamma_{i}\right)} \quad \text { or } \quad \Gamma_{\gamma_{i}},[\perp]^{-s\left(\gamma_{i}\right)} \triangleleft \Delta_{\gamma_{i}},
$$

where $\Gamma_{\gamma_{i}}=\left[P\left(\varphi_{i 1}\right)\right]^{a_{i 1}}, \ldots,\left[P\left(\varphi_{i m_{i}}\right)\right]^{a_{i m_{i}}}$ and $\Delta_{\gamma_{i}}=$ $\left[P\left(\varphi_{i m_{i}+1}\right)\right]^{a_{i m_{i+1}}}, \ldots,\left[P\left(\varphi_{i n_{i}}\right)\right]^{a_{i n_{i}}}$.

Assume that $\gamma_{i}:=\sum_{k=1}^{n_{i}} a_{i k} P\left(\varphi_{i k}\right) \geq c_{i}$ and hence $\gamma_{i}^{H}$ is of the first form above, with $\leq$ in place of $\triangleleft$. We have that $\mathbf{M}$ is a model of $\left(\gamma_{i}\right)^{H}$ if and only if

$$
\begin{aligned}
& a_{i 1}\left(\mu\left(\varphi_{i 1}^{\mathbf{M}}\right)-1\right)+\cdots+a_{i m_{i}}\left(\mu\left(\varphi_{i m_{i}}^{\mathrm{M}}\right)-1\right) \leq \\
& a_{i m_{i}+1}\left(\mu\left(\varphi_{i m_{i+1}}^{\mathbf{M}}\right)-1\right)+\cdots+a_{i n_{i}}\left(\mu\left(\varphi_{i n_{i}}^{\mathbf{M}}\right)-1\right)-s\left(\gamma_{i}\right)
\end{aligned}
$$

which in turn holds iff

$$
\begin{aligned}
\sum_{k=1}^{m_{i}} a_{i k} \mu\left(\varphi_{i m_{i}}^{\mathrm{M}}\right)-\sum_{k=1}^{m_{i}} a_{i k} \leq & \sum_{k=m_{i}+1}^{n_{i}} a_{i k} \mu\left(\varphi_{i k}^{\mathbf{M}}\right)-\sum_{k=m_{i}+1}^{n_{i}} a_{i k} \\
& -\left(\sum_{k=1}^{m_{i}} a_{i k}-\sum_{k=m_{i}+1}^{n_{i}} a_{i k}+c\right) .
\end{aligned}
$$


It is now easy to check that the latter holds iff $\sum_{k=1}^{n_{i}} a_{i k}\left(\varphi_{i k}\right)^{\mathbf{M}} \geq c_{i}$, i.e. $\left\|\gamma_{i}\right\|_{\mathbf{M}}=1$. Other cases are similar.

By classical reasoning, since $\mathbf{M}$ is a model of $\gamma_{i}$ if and only if it is a model of $\gamma_{i}^{H}$, the same will hold for $\gamma_{1} \vee \ldots \vee \gamma_{n}$ and $\gamma_{1}^{H}|\ldots| \gamma_{n}^{H}$. This concludes the proof.

Lemma 3. Let $\delta$ be a clause in $\mathcal{L}^{\operatorname{Pr}_{\text {lin }}}$ such that $\models_{\mathrm{HPr}_{\mathrm{E}}} \delta^{H}$. Then $\vdash_{\operatorname{Pr}_{\text {lin }}} \delta$.

Proof. Let $V$ be the set of variables in $\delta$. Hence, due to Lemma 1, $\vdash_{\text {HE }}\left(\delta^{H}\right)^{*} \mid A X_{V}^{*}$. Note that, by the definition of the translations $(\cdot)^{H}$ and $(\cdot)^{*}$, and of the set $A X_{V}^{*}$, the hypersequent $\left(\delta^{H}\right)^{*} \mid A X_{V}^{*}$ only contains propositional variables.

Hence we can find, for both $\left(\delta^{H}\right)^{*}$ and $A X_{V}^{*}$, an antiimage of the (composition of the) translations $(\cdot)^{H}$ and $(\cdot)^{*}$. For $\left(\delta^{H}\right)^{*}$ this is obviously the formula $\delta$, while for $A X_{V}^{*}$, we let $\alpha$ be the clause in $\operatorname{Pr}_{\text {lin }}$ such that $\left(\alpha^{H}\right)^{*}=A X_{V}^{*}$.

We now prove that, for each hypersequent occurring in a derivation of $\left(\delta^{H}\right)^{*} \mid A X_{V}^{*}$ in HŁ, the anti-image of the (composition of the) translations $(\cdot)^{*}$ and $(\cdot)^{H}$ is derivable in $\operatorname{Pr}_{l i n}$. When applied to the root of the derivation, this shows $\vdash_{\operatorname{Pr}_{l i n}} \delta \vee \alpha$. We proceed by induction on the length of the derivation.

The case of axioms is easy. For the rules (ew), (ec), derivations in classical logic suffice. Let us consider the case where the last applied rule is $\left(\right.$ split $\left._{\leq}\right)$, with premise $G \mid \Gamma_{1}, \Gamma_{2} \leq \Delta_{1}, \Delta_{2}$ and conclusion $G \mid \Gamma_{1} \leq$ $\Delta_{1} \mid \Gamma_{2} \leq \Delta_{2}$. Let $\gamma$ and $\varepsilon_{1,2}$ be formulas such that $\left(\gamma^{H}\right)^{*}=G$ and $\left(\varepsilon_{1,2}^{H}\right)^{*}=\Gamma_{1}, \Gamma_{2} \leq \Delta_{1}, \Delta_{2}$. By induction hypothesis, $\vdash_{\operatorname{Pr}_{l i n}} \gamma \vee \varepsilon_{1,2}$.

Without loss of generality, let $\varepsilon_{1}:=\left(\sum_{i=1}^{n_{1}} a_{i} P\left(\varphi_{i}\right) \geq\right.$ $\left.c_{1}\right)$ be such that $\left(\varepsilon_{1}^{H}\right)^{*}=\Gamma_{1} \leq \Delta_{1}$, and $\varepsilon_{2}:=$ $\left(\sum_{j=n_{1}+1}^{n_{2}} a_{j} P\left(\varphi_{j}\right) \geq c_{2}\right)$ such that $\left(\varepsilon_{2}^{H}\right)^{*}=\Gamma_{2} \leq \Delta_{2}$. We have then $\varepsilon_{1,2}:=\left(\sum_{i=1}^{n_{2}} a_{i} P\left(\varphi_{i}\right) \geq c_{1}+c_{2}\right)$. We want to prove that $\vdash_{\operatorname{Pr}_{l i n}} \gamma \vee \varepsilon_{1} \vee \varepsilon_{2}$. First, we need to show that

$$
\begin{array}{r}
\vdash_{\operatorname{Pr}_{l i n}} \sum_{i=1}^{n_{1}} a_{i} P\left(\varphi_{i}\right)>c_{1} \wedge \\
\sum_{j=n_{1}+1}^{n_{2}} a_{j} P\left(\varphi_{j}\right)>c_{2} \rightarrow \\
\rightarrow \sum_{i=1}^{n_{2}} a_{i} P\left(\varphi_{i}\right)>c_{1}+c_{2} .
\end{array}
$$

This follows by suitable applications of (LQ6), (LQ7), (LQ4). The derivation of $\vdash_{\operatorname{Pr}_{l i n}} \gamma \vee \varepsilon_{1} \vee \varepsilon_{2}$ is then obtained by classical reasoning.

For the other structural rules we proceed similarly. Note that we do not need to check the case of logical rules, since they cannot occur in a derivation of $\left(\delta^{H}\right)^{*} \mid A X_{V}^{*}$. Indeed, the latter hypersequent only contains propositional variables, and all the rules in HE are analytic, i.e. their premises can only contain subformulas of the conclusion.

Thus, we have proved $\vdash_{\operatorname{Pr}_{l i n}} \delta \vee \alpha$. If we show that $\vdash \operatorname{Pr}_{l i n} \neg \alpha$, the proof is finished. Let us recall the structure of $\alpha$. Some of its disjuncts correspond to sequents in $R_{V}$, hence they are formulas of the kind $P(\varphi)<1$, where $\varphi$ is a classical tautology. Their negation, i.e. $P(\varphi) \geq 1$, can be proved in $\operatorname{Pr}_{\text {lin }}$, since $\varphi \leftrightarrow \top$, by (QU2) $P(\top)=1$ and by (QUGEN) $P(\varphi)=P(\top)$. The remaining disjuncts come from the hypersequents in $\left(A_{V}^{1}\right)-\left(A_{V}^{3}\right)$, hence they are formulas of the kind:

$$
\begin{aligned}
\alpha_{1}: & =P(\psi)<P(\varphi)+P(\varphi \rightarrow \psi) \\
\alpha_{2}:= & (1<P(\varphi)+P(\neg \varphi)) \vee(P(\varphi)+P(\neg \varphi)<1) \\
\alpha_{3}:= & (P(\varphi)+P(\psi)<P(\varphi \vee \psi)+P(\varphi \wedge \psi)) \vee \\
& \quad \vee(P(\varphi \vee \psi)+P(\varphi \wedge \psi)<P(\varphi)+P(\psi))
\end{aligned}
$$

The negation of each one of such formulas corresponds to a basic fact about probability, which is derivable in $\operatorname{Pr}_{\text {lin }}$ : in particular showing $\neg \alpha_{1}$ amounts to showing a derivation of the formula $P(\psi) \geq P(\varphi)+P(\neg \varphi \vee \psi)$, showing $\neg \alpha_{2}$ amounts to $P(\neg \varphi)+P(\varphi)=1$ and $\neg \alpha_{3}$ to $P(\varphi \vee \psi)+P(\varphi \wedge \psi)=P(\varphi)+P(\psi)$.

Let us sketch the proof of the last formula as an example. By the axiom (QU3), we have $P((\varphi \vee \psi) \wedge \varphi)+$ $P((\varphi \vee \psi) \wedge \neg \varphi)=P(\varphi \vee \psi)$, which is equivalent to $P(\varphi)+P(\neg \varphi \wedge \psi)=P(\varphi \vee \psi)$. On the other hand, again by (QU3), we have $P(\psi)=P(\psi \wedge \neg \varphi)+P(\varphi \wedge \psi)$. The derivation of $\neg \alpha_{3}$ then follows from the last two formulas we derived and a simple manipulation of equalities.

Using the last two lemmata we can formulate the following faithful translation of $\operatorname{Pr}_{l i n}$ into $\mathrm{HPr}_{€}$ and the promised alternative proof of axiomatization of $\operatorname{Pr}_{l i n}$.

Theorem 3. Let $\Gamma \cup\{\delta\}$ be a finite set of formulas of $\mathcal{L}^{\operatorname{Pr}_{\text {lin }}}$ and $\delta_{1} \wedge \cdots \wedge \delta_{m}$ a conjunctive normal form of $\left(\bigwedge_{\gamma \in \Gamma} \gamma\right) \rightarrow \delta$. Then, $\Gamma \vdash_{\operatorname{Pr}_{l i n}} \delta$ iff $\Gamma \models \operatorname{Pr}_{\text {lin }} \delta$ iff $=\operatorname{HPr}_{\varepsilon} \delta_{i}^{H}$ for each $i$.

Proof. The first implication is just soundness. The second one follows from Lemma 2 (as $\Gamma \models_{\operatorname{Pr}_{l i n}} \delta$ clearly implies $\models \operatorname{Pr}_{l i n} \delta_{i}$ for each $i$ ). The final one follows from Lemma 3 and the fact that $\vdash_{\operatorname{Pr}_{l i n}} \delta_{i}$ for each $i$, we obtain $\Gamma \vdash_{\operatorname{Pr}_{l i n}} \delta$ using a classical reasoning.

Finally, we can use our last result to provide the promised alternative translation from $\operatorname{Pr}_{l i n}$ into $\operatorname{Pr}_{\mathfrak{E}_{\triangle}}$. The key is to interpret any $\operatorname{Pr}_{€}$-hypersequent $G$ (using again essentially McNaughton theorem, adapting from [16]) as a formula $I(G)$ of $\mathrm{Pr}_{\mathrm{七}_{\triangle}}$ such that $=_{\mathrm{HPr}_{\mathrm{E}}} G$ if and only if $=_{\operatorname{Pr}_{\mathrm{E}_{\triangle}}} I(G)$. The rest of the proof is a simple application of the previous theorem. 
Theorem 4. Let $\Gamma \cup\{\delta\}$ be a finite set of formulas of $\mathcal{L}^{\operatorname{Pr}_{\text {lin }}}$ and $\delta_{1} \wedge \cdots \wedge \delta_{m}$ a conjunctive normal form of $\left(\bigwedge_{\gamma \in \Gamma} \gamma\right) \rightarrow \delta$. Then, $\Gamma \models_{\operatorname{Pr}_{l i n}} \delta$ if and only if ${ }={\mathrm{P \textrm {P } _ { \mathrm { E } }}}_{\Delta} I\left(\delta_{1}^{H}\right) \wedge \cdots \wedge I\left(\delta_{m}^{H}\right)$.

\section{Conclusion}

We have presented several faithful translations between logics of uncertainty. Namely, we have translated $\operatorname{Pr}_{l i n}$ into $\operatorname{HPr}_{\mathrm{E}}$ and (in two different ways) into $\operatorname{Pr}_{E_{\Delta}}$, and we have translated $\operatorname{Pr}_{\text {pol }}$ into $\operatorname{Pr}_{\mathrm{PE}_{\Delta}}$.

The translations we have presented showcase the power of the many-valued semantics. The logic $\operatorname{Pr}_{l i n}$ has indeed a complex syntax (with many constants for numbers) to express inequalities involving probabilities of events, and needs explicit axioms to manipulate such linear inequalities, while $\mathrm{Pr}_{\mathrm{E}_{\Delta}}$ can instead directly express such inequalities thanks to its semantics. Note that our second syntactic translation provides, as an interesting corollary, an alternative proof of completeness for the logic $\operatorname{Pr}_{\text {lin }}$. Moreover, our translation of $\mathrm{Pr}_{p o l}$ has given it an indirect axiomatization.

We believe that translations of other logics of uncertainty are likely to bring similar benefits. Let us indicate some concrete directions for further research. First, we will study in our framework the systems introduced in $[4,12]$, which allow for modal formulas like $P_{\geq r} \varphi$ standing for "the probability of $\varphi$ is at least $r$ " where $r$ is a constant for a rational number and binary modalities expressing that a formula provides probabilistic confirmation or disconfirmation for another. Another important research direction is to provide analytic calculi for logics of uncertainty in the literature, where so far little is known (see e.g. [15]). Indeed, we plan to suitably extend the hypersequent calculus HE discussed here, to obtain an analytic system for the logic $\operatorname{Pr}_{£}$, and investigate whether such framework can be extended and applied to other logics of uncertainty.

\section{Acknowledgement}

The authors were supported by the grant GA1704630S of the Czech Science Foundation. Petr Cintula and Paolo Baldi also acknowledge support by RVO 67985807 .

\section{References}

[1] S. Aguzzoli, S. Bova, B. Gerla, Free algebras and functional representation for fuzzy logics, in: Cintula et al. [2], pp. 713-791.

[2] P. Cintula, P. Hájek, C. Noguera (Eds.), Vol. 3738 of Studies in Logic, Mathematical Logic and Foundations, College Publications, London, 2011.
[3] P. Cintula, C. Noguera, Modal logics of uncertainty with two-layer syntax: A general completeness theorem, in: U. Kohlenbach, P. Barceló, R. de Queiroz (Eds.), Logic, Language, Information, and Computation, Springer, 2014, pp. 124136.

[4] D. Doder, Z. Ognjanovic, Probabilistic logics with independence and confirmation, Studia Logica 105 (5) (2017) 943-969.

[5] R. Fagin, J.Y. Halpern, Reasoning about knowledge and probability, J. ACM 41 (2) (1994) 340367.

[6] R. Fagin, J. Y. Halpern, N. Megiddo, A logic for reasoning about probabilities, Information and Computation 87 (1-2) (1990) 78-128.

[7] T. Flaminio, L. Godo, E. Marchioni, Reasoning about uncertainty of fuzzy events: An overview, in: P. Cintula, C. Fermüller, L. Godo (Eds.), Understanding Vagueness: Logical, Philosophical, and Linguistic Perspectives, College Publications, 2011, pp. 367-400.

[8] P. Hájek, Metamathematics of Fuzzy Logic, Vol. 4 of Trends in Logic, Kluwer, Dordrecht, 1998.

[9] P. Hájek, L. Godo, F. Esteva, Fuzzy logic and probability, in: Proceedings of the 11th Annual Conference on Uncertainty in Artificial Intelligence UAI '95, 1995, pp. 237-244.

[10] P. Hájek, D. Harmancová, Medical fuzzy expert systems and reasoning about beliefs, in: P. Barahona, M. Stefanelli, J. Wyatt (Eds.), Artificial Intelligence in Medicine, 1995, pp. 403-404.

[11] J.Y. Halpern, Reasoning About Uncertainty, MIT Press, 2005.

[12] A. Heifetz, P. Mongin, Probability logic for type spaces, Games and Economic Behavior 35 (1) (2001) 31-53.

[13] A. Horn, A. Tarski, Measures on Boolean algebras, Transactions of American Mathematical Society 64 (1948) 467-497.

[14] T. Kroupa, Representation and extension of states on MV-algebras, Archive for Mathematical Logic 45 (4) (2006) 381-392.

[15] C. Kupke, D. Pattinson, On modal logics of linear inequalities, in: Advances in Modal Logic 8, 2010, pp. $235-255$.

[16] G. Metcalfe, N. Olivetti, D. M. Gabbay, Proof Theory for Fuzzy Logics, Springer, 2008. 